Int. J. Morphol.,

35(4):1547-1552, 2017.

\title{
Asociación entre Índice de Masa Corporal y Depresión en Mujeres Adolescentes
}

\author{
Association Between Body Mass Index and Depression in Adolescent Women
}

Juan Ocampo ${ }^{1,2}$; Marco Guerrero' ${ }^{1,3}$; Luis Espín ${ }^{1,3}$; Clivia Guerrero ${ }^{1,4,5}$ \& Romel Aguirre ${ }^{6}$

OCAMPO, J.; GUERRERO, M.; ESPÍN, L.; GUERRERO, C. \& AGUIRRE, R. Asociación entre índice de masa corporal y depresión en mujeres adolescentes de la ciudad de Quito, Ecuador. Int. J. Morphol., 35(4):1547-1552, 2017.

RESUMEN: El sobrepeso y la obesidad tienen una prevalencia que está aumentando mundialmente, principalmente en adolescentes; se relacionan con enfermedades crónicas graves y depresión, especialmente en mujeres. Se estudió a 180 mujeres adolescentes de Quito, Ecuador, muestreadas por clusters bietápico, a quienes se midió el índice de masa corporal (IMC) y se realizó el Inventario de Depresión de Beck I-A (IDB I-A), luego de obtener el asentimiento y consentimiento informado. Los datos fueron ingresados en Excel 2011 y procesados en SPSS v22, para determinar la asociación entre el IMC y depresión. La validación interna del IDB obtuvo un Alfa de Cronbach de 0,818; para la variable respuesta se utilizó la prueba de ANOVA de un factor, con un error alfa <0,05; se utilizó la prueba post-hoc de Games-Howell para diferenciar las medias; se obtuvieron datos descriptivos de posición y dispersión de las variables. Se encontró un 27,22 \% de individuos con sobrepeso y obesidad y un $25,56 \%$ con problemas depresivos, más frecuentes en mujeres de 19 años. Hubo diferencia entre las medias de depresión del grupo de peso normal vs sobrepeso y obesidad ( $\mathrm{p}<0,05)$; la prueba post-hoc de Games-Howell diferenció peso normalsobrepeso $(\mathrm{p}<0,05)$. El riesgo relativo fue de 8,55. Estos resultados son similares, tanto en las variables de sobrepeso y obesidad, como en depresión, a los informados en otros estudios. Se observó una asociación estadísticamente significativa entre las variables de estudio (sobrepesoobesidad y depresión), en mujeres adolescentes de la ciudad de Quito, Ecuador.

PALABRAS CLAVE: Índice de masa corporal; Obesidad; Depresión; Inventario de Depresión de Beck; Adolescentes.

\section{INTRODUCCIÓN}

La identificación de sobrepeso y obesidad es comúnmente realizada por medio del índice de masa corporal (IMC), que se define como la medida del peso (masa) del sujeto, corregida por el cuadrado de la estatura (Lacunza et al., 2015). El IMC es una herramienta valiosa en la determinación del grado de sobrepeso y obesidad en la población adolescente, aún mejor que el porcentaje de grasa corporal, el cual puede sobreestimar estas condiciones (Aguilar Cordero et al., 2012; Padilla, 2014). No obstante, debe considerarse la diferencia de los niveles umbrales de IMC que indican sobrepeso y obesidad en adolescentes, en concordancia con las recomendaciones de la Organización Mundial de la Salud (OMS) y de su Grupo de Trabajo Internacional sobre Obesidad (IOTF, por sussiglas en inglés); desde los 18 años en adelante estos niveles son los mismos que los de la población adulta (Kaufer-Horwitz \& Toussaint, 2008). La tipificación ponderal, de acuerdo a los niveles del IMC, con- templa las categorías de Peso Bajo, Peso Normal, Sobrepeso y Obesidad (Lacunza et al., 2015; Ramos Rosales et al., 2016).

Según la OMS, la obesidad es un problema de salud pública extremadamente importante y es considerada la nueva epidemia planetaria del siglo XXI (Cañoles et al., 2015). El sobrepeso y la obesidad se definen como la acumulación anormal o excesiva de grasa que puede perjudicar la salud; esta última se relaciona con la aparición de un gran número de enfermedades crónicas, como la diabetes mellitus, hipertensión, dislipidemias, enfermedad cardiovascular, esteatohepatitis no alcohólica, artrosis, algunos tipos de neoplasias, entre otras (Aguilar Cordero et al.).

A pesar de estos peligros, a nivel mundial el $39 \%$ de personas de 18 o más años tiene sobrepeso y el $13 \%$, obesidad; la proyección es alarmante: para el año 2030 la prevalen-

\footnotetext{
${ }^{1}$ Programa de Doctorado en Ciencias Morfológicas, Universidad de La Frontera, Temuco, Chile.

${ }^{2}$ Profesor de la Cátedra de Histología Normal. Carrera de Medicina. Universidad Central del Ecuador, Quito, Ecuador.

${ }^{3}$ Profesor de la Cátedra de Anatomía Normal. Carrera de Medicina. Universidad Central del Ecuador, Quito, Ecuador.

${ }^{4}$ Profesor de la Cátedra de Medicina Legal. Carrera de Obstetricia. Universidad Central del Ecuador, Quito, Ecuador.

${ }^{5}$ Médico Legista. Departamento Médico Legal de la Policía Judicial de Pichincha. Policía Nacional del Ecuador.

${ }^{6}$ Psicólogo. Profesor de la Cátedra de Psicología Médica. Carrera de Medicina. Universidad Central del Ecuador, Quito, Ecuador.
} 
cia de sobrepeso y de obesidad serán de 89 y $48 \%$, respectivamente (Quintero et al., 2016). Su causa es un desequilibrio entre la ingesta y el gasto de calorías, coadyuvado por la constante disponibilidad de alimentos con alto contenido energético y/o el déficit de actividades físicas (Ramos Rosales et al.).

Así, la prevalencia de obesidad, en lugar de controlarse y reducirse, está aumentando en todas las edades, y el grupo etario con la mayor tasa de incremento es el de los adolescentes (Calderón et al., 2010). En España, las cifras de obesidad en adolescentes preocupan, pues uno de cada tres presenta sobrepeso y, uno de cada veinte, obesidad; esta es una de las cifras más altas de Europa (Aguilar Cordero et $a l$. .). En Argentina (Buenos Aires) se plantea un 15,3\% de mujeres adolescentes con sobrepeso y un 3,5\%, con obesidad (Lacunza et al., 2013). En Ecuador, el exceso de peso y la obesidad afectan al 24,5\%, de los adolescentes entre 15 y 19 años (Freire et al., 2014), lo que es más común en mujeres $(21,5 \%)$ que en hombres (20,8 \%), (Yépez et al., 2008). $\mathrm{Si}$ a esto se suma la probabilidad de que el $80 \%$ de adolescentes obesos mantengan esta condición en la adultez, la situación se agrava aún más, pues mientras mayor edad tenga el individuo, tendrá más posibilidades de complicaciones patológicas (Ponce et al., 2014).

Además, el sobrepeso y la obesidad presentan relación con algunas alteraciones psicológicas, como la depresión (Cañoles et al.). La depresión es una anomalía de la afectividad, quizás la más frecuente entre los adolescentes de todo el mundo; es un trastorno del estado de ánimo que conlleva a la incapacidad del individuo para interesarse y disfrutar de las cosas, con múltiples consecuencias, como la ideación suicida y el suicidio consumado entre el 15 y el $20 \%$ de los que la padecen; la depresión puede desencadenar conducta antisocial, consumo de tabaco, alcohol y otras drogas y actúa como factor de riesgo en el desarrollo de cualquier otro tipo de psicopatología (Beltrán et al., 2012). Existe una relación inversa entre la obesidad y la autoestima y una directa respecto a la depresión; asimismo, se identifica que las mujeres adolescentes están más afectadas que los hombres, debido a la cultura social (Ceja Espíritu et al., 2010).

Según Trejo Ortiz et al. (2010) y Fernández-Bustos et al. (2015), en la etapa adolescente, el autoconcepto físico es muy importante pues influye decisivamente en la estructuración del autoconcepto general; el sobrepeso y la obesidad generan insatisfacción con la imagen corporal; así, las adolescentes con mayor IMC son las más susceptibles a sentirse insatisfechas. La autoestima es un factor importante en la predicción de síntomas depresivos en adultos jóvenes; mientras que los hombres evalúan su cuerpo en base de su fuerza, las mujeres lo hacen según la delgadez y la forma de ciertas partes del mismo (Ortega Becerra et al., 2015).
También se evidencian cambios significativos en niveles de ansiedad y depresión en mujeres jóvenes, tras exponerlas a imágenes publicitarias que pretenden brindar estereotipos de belleza femenina y actitudes de alimentación, cambios que son mayores conforme aumenta el IMC de ellas (Jiménez-Cruz \& Silva-Gutiérrez, 2008).

Por otro lado, existe una mayor prevalencia de trastornos alimentarios, como el comer emocional, principalmente en mujeres adolescentes, lo que constituye un predictor confiable de sobrepeso (Camarillo et al., 2013; Ramos Rosales et al.). Las adolescentes con sobrepeso y obesidad tienen conductas de control de peso más riesgosas e injuriantes, como dietas extremas, generalmente sin supervisión profesional, acompañadas de la insatisfacción corporal y/u obsesión por la delgadez, que puede conducir a la depresión, común en estas edades (Contreras et al., 2015). Es importante recomendar actividad física vigorosa en la población, pues según Martínez Rojano et al. (2014) constituye un mediador importante que atenúa la fuerza de la asociación entre IMC elevado y depresión.

La teoría de la depresión de Beck explica cognitivamente esta enfermedad, que se basa en un modelo de labilidad al estrés, con esquemas distorsionados de pensamiento que influencian negativamente en lo que el individuo percibe, codifica e interpreta sobre sí mismo, sobre el mundo y sobre el futuro; a partir de esta explicación surgió el Inventario de Depresión de Beck (IBD), para detectar síntomas depresivos y su gravedad, el cual es muy utilizado en estudios sobre población adolescente clínica y no clínica, por sus adecuadas propiedades psicométricas y su facilidad de ejecución (Beltrán et al.).

En consideración a la vigencia y peligrosidad de los antecedentes señalados y a la escasez de estudios realizados en esta área, tanto en Ecuador como en América Latina, se ha realizado este trabajo de investigación con el objetivo de determinar la asociación entre el IMC y depresión, medida por el IDB I-A, en mujeres adolescentes de la ciudad de Quito, Ecuador.

\section{MATERIAL Y MÉTODO}

Se realizó un estudio epidemiológico, observacional y transversal en 180 mujeres adolescentes de 14 a 19 años de edad, seleccionadas aleatoriamente, entre estudiantes de las unidades educativas del Distrito Metropolitano de Quito (DMQ), mediante muestreo bietápico por clusters y aleatorio simple (muestra representativa de la población de mujeres adolescentes del DMQ), a quienes, posterior a la aprobación del comité de ética de la Universidad Central del 
Ecuador, el asentimiento y consentimiento informado (según la edad), se les sometió a la medición del peso en kilogramos y talla en centímetros, mediante técnicas antropométricas estandarizadas, en una balanza-tallímetro Health o Meter® (USA). Además, se les aplicó el cuestionario del IDB-IA, mediante entrevista personal. Las mediciones y las encuestas fueron realizadas por los investigadores.

Posteriormente, se trasladaron los datos a una hoja electrónica de Excel 2011 para su depuración y luego fueron procesados en el programa estadístico SPSS v22. Se establecieron tres categorías para el IMC, y los niveles de corte para la determinación de los grupos se realizaron de conformidad a las recomendaciones de la IOTF-OMS, según las edades.

Los ítems del cuestionario del IDB-IA fueron sometidos a validación interna mediante la aplicación del Alfa de Cronbach, el cual dio un valor de 0,818 . Luego se sometió la variable respuesta a prueba estadística según el factor IMC, mediante ANOVA de un factor, estableciendo como error alfa un valor $<0,05$. Para diferenciar las medias de cada gru- po se utilizó la prueba post-hoc de Games-Howell, en razón de que las varianzas resultantes no fueron homogéneas. Se obtuvo también los datos descriptivos de posición y dispersión de las variables antes mencionadas.

\section{RESULTADOS}

En el estudio efectuado encontramos que un 27,22 $\%$ de la población de mujeres adolescentes presentó sobrepeso y obesidad (IC $95 \%$ 20,72-33,72). El grupo de 19 años de edad mostró una mayor frecuencia. La distribución del estado nutricional por IMC, según edad, se presenta en la Tabla I.

Asimismo, el 25,56 \% de la población estudiada de mujeres adolescentes mostró desde leve perturbación del estado de ánimo hasta depresión grave (IC $95 \%$ 19,1831,93). A los 19 años de edad se observó una mayor presencia de estas alteraciones. La Tabla II muestra la distribución de los resultados del IDB-IA por edad.

Tabla I. Distribución de frecuencias del estado nutricional por índice de masa corporal, según edad, en mujeres adolescentes del Distrito Metropolitano de Quito, Ecuador, 2016.

\begin{tabular}{|c|c|c|c|c|c|c|c|c|}
\hline \multirow{3}{*}{$\begin{array}{l}\text { Edad } \\
\text { en años }\end{array}$} & \multicolumn{6}{|c|}{ Estado Nutricional } & \multirow{2}{*}{\multicolumn{2}{|c|}{ Total }} \\
\hline & \multicolumn{2}{|c|}{ Peso normal } & \multicolumn{2}{|c|}{ Sobrepeso } & \multicolumn{2}{|c|}{ Obesidad } & & \\
\hline & $\mathrm{n}$ & $\%$ & $\mathrm{n}$ & $\%$ & $\mathrm{n}$ & $\%$ & $\mathrm{n}$ & $\%$ \\
\hline 14 & 25 & 13,89 & 5 & 2,78 & 0 & 0,00 & 30 & 16,67 \\
\hline 15 & 18 & 10,00 & 6 & 3,33 & 2 & 1,11 & 26 & 14,44 \\
\hline 16 & 19 & 10,56 & 4 & 2,22 & 1 & 0,56 & 24 & 13,33 \\
\hline 17 & 18 & 10,00 & 6 & 3,33 & 2 & 1,11 & 26 & 14,44 \\
\hline 18 & 30 & 16,67 & 6 & 3,33 & 2 & 1,11 & 38 & 21,11 \\
\hline 19 & 21 & 11,67 & 14 & 7,78 & 1 & 0,56 & 36 & 20,00 \\
\hline Total & 131 & 72,78 & 41 & 22,78 & 8 & 4,44 & 180 & 100,00 \\
\hline
\end{tabular}

Según cortes de IMC en adolescentes, recomendados por la OMS y su Grupo de Trabajo Internacional sobre Obesidad (IOTF, por sus siglas en inglés).

Tabla II. Distribución de frecuencias del Inventario de Depresión de Beck I-A, según edad, en mujeres adolescentes del Distrito Metropolitano de Quito, Ecuador, 2016.

\begin{tabular}{|c|c|c|c|c|c|c|c|c|c|c|}
\hline \multirow{3}{*}{$\begin{array}{l}\text { Edad en } \\
\text { años }\end{array}$} & \multicolumn{8}{|c|}{ Inventario de Depresión de Beck I-A } & \multirow{2}{*}{\multicolumn{2}{|c|}{ Total }} \\
\hline & \multicolumn{2}{|c|}{ Normal } & \multicolumn{2}{|c|}{$\begin{array}{c}\text { Leve perturbación } \\
\text { de e stado de } \\
\text { ánimo }\end{array}$} & \multicolumn{2}{|c|}{$\begin{array}{l}\text { Depresión } \\
\text { moderada }\end{array}$} & \multicolumn{2}{|c|}{ Depresión grave } & & \\
\hline & $\mathrm{n}$ & $\%$ & $\mathrm{n}$ & $\%$ & $\mathrm{n}$ & $\%$ & $\mathrm{n}$ & $\%$ & $\mathrm{n}$ & $\%$ \\
\hline 14 & 22 & 12,22 & 6 & 3,33 & 1 & 0,56 & 1 & 0,56 & 30 & 16,67 \\
\hline 15 & 17 & 9,44 & 5 & 2,78 & 4 & 2,22 & 0 & 0,00 & 26 & 14,44 \\
\hline 16 & 18 & 10,00 & 3 & 1,67 & 3 & 1,67 & 0 & 0,00 & 24 & 13,33 \\
\hline 17 & 20 & 11,11 & 3 & 1,67 & 3 & 1,67 & 0 & 0,00 & 26 & 14,44 \\
\hline 18 & 31 & 17,22 & 3 & 1,67 & 3 & 1,67 & 1 & 0,56 & 38 & 21,11 \\
\hline 19 & 26 & 14,44 & 5 & 2,78 & 5 & 2,78 & 0 & 0,00 & 36 & 20,00 \\
\hline Total & 134 & 74,44 & 25 & 13,89 & 19 & 10,56 & 2 & 1,11 & 180 & 100,00 \\
\hline
\end{tabular}

Estado afectivo según puntaje de IDB I-A: 1-10 Normal; 11-16 Leve perturbación del estado de ánimo; 17-20 Estados de depresión intermitentes; 21-30 Depresión moderada; 31-40 Depresión grave; +40 Depresión extrema. 
Tabla III. Distribución de valores de posición y dispersión del Inventario de Depresión de Beck I-A, según estado nutricional en mujeres adolescentes del Distrito Metropolitano de Quito, Ecuador, 2016.

\begin{tabular}{|c|c|c|c|c|}
\hline \multicolumn{5}{|c|}{ Inventario de Depresión de Beck I-A } \\
\hline & & Peso Normal & Sobrepeso & Obesidad \\
\hline \multirow[t]{2}{*}{ Media } & & 7,17 & 12,05 & 12,88 \\
\hline & Límite inferior & 6,28 & 9,32 & 6,72 \\
\hline IC $95 \%$ para la media & Límite superior & 8,06 & 14,77 & 19,03 \\
\hline Mediana & & 6 & 10 & 11,5 \\
\hline Varianza & & 26,65 & 74,60 & 54,13 \\
\hline Desviación estándar & & 5,16 & 8,64 & 7,36 \\
\hline Mínimo & & 0 & 1 & 4 \\
\hline Máximo & & 38 & 32 & 21 \\
\hline Rango intercuartil & & 6 & 17 & 16 \\
\hline Error estándar de la me & edia & 0,45 & 1,35 & 2,60 \\
\hline Coeficiente de variació & & 71,97 & 71,70 & 57,14 \\
\hline
\end{tabular}

Estado afectivo según puntaje de IDB I-A: 1-10 Normal; 11-16 Leve perturbación del estado de ánimo; 17-20 Estados de depresión intermitentes; 21-30 Depresión moderada; 31-40 Depresión grave; +40 Depresión extrema.

Encontramos una diferencia estadísticamente significativa entre las medias del IDB-IA del grupo de peso normal, por una parte, y de sobrepeso y obesidad, por la otra $(\mathrm{p}<0,05)$, corroborado por la prueba post-hoc de Games-Howell, que comparó peso normal-sobrepeso $(\mathrm{p}<0,05)$. Los valores de posición y dispersión del IDB I-A se muestran en la Tabla III.

$\mathrm{Al}$ dicotomizar las variables de estudio se obtuvo un valor de riesgo relativo de 8,55 (IC $95 \%$ 3,90-18,78).

\section{DISCUSIÓN}

En Ecuador se evidencia, a lo largo del tiempo, una tendencia general de la población hacia el sobrepeso y la obesidad. El estudio realizado por Yépez et al. encontró una prevalencia de sobrepeso y obesidad de $21,5 \%$ en mujeres adolescentes ecuatorianas. Freire et al. llevó a cabo la encuesta nacional de nutrición (ENSANUT), durante el año 2012 , en cuya base de datos se puede evidenciar una prevalencia de $24,8 \%$ de sobrepeso y obesidad en las mujeres adolescentes quiteñas de 14 a 19 años de edad. Si comparamos estos con los resultados de nuestro estudio, que fue ligeramente superior al $27 \%$ de la muestra, claramente observamos una tendencia creciente de esta parte de la población hacia el sobrepeso y la obesidad pues han crecido 5,72 puntos porcentuales desde 2008, de acuerdo a lo señalado por Yépez et al. Por otra parte, nuestras cifras son comparables con las reportadas en otros países de Latinoamérica, como México, donde se indicó una prevalencia de sobrepeso y obesidad en el $27,3 \%$ de las mujeres universitarias con una edad promedio de 18,5 años (Ríos Saldaña \& Osornio Castillo, 2014).
En lo que concierne a la depresión, un estudio retrospectivo de 456 casos de adolescentes, realizado en el Hospital Psiquiátrico Lorenzo Ponce de Guayaquil, Ecuador, Morla Boloña et al. (2006) encontraron una prevalencia de los diferentes niveles de depresión, según el CIE-10, de $22,9 \%$ (100 casos), de los cuales el $75 \%$ eran mujeres. Nuestro estudio reflejó un 25,56\% de prevalencia de depresión, en todos sus niveles, y se presentó mayoritariamente en las mujeres de 19 años de edad, lo cual coincide con los mayores índices de sobrepeso y obesidad. Esto podría estar relacionado, además del mayor IMC, con la transición desde la etapa estudiantil de secundaria al enfrentamiento de nuevos retos sociales, como los universitarios y/o laborales.

Respecto a la asociación del sobrepeso y de la obesidad, con la depresión, fue estadísticamente significativa en nuestra investigación. Estos resultados fueron similares al realizado por Miranda (2013), en mujeres de Santiago de Chile, quien encontró una relación directa entre sobrepesoobesidad con depresión, según los criterios diagnósticos del CIE10. Esta asociación podría explicarse por el deterioro de la imagen corporal (Trejo Ortiz et al.), y del autoconcepto físico y general (Fernández-Bustos et al.), que conducen, a las personas con un IMC elevado, a la depresión; y a los trastornos de la alimentación relacionados (Calderón et al.); todas estas alteraciones se presentaron más intensamente en mujeres y en los años de adolescencia.

La asociación entre sobrepeso-obesidad y el síndrome depresivo en mujeres adolescentes quiteñas fue consistente; nuestros resultados demostraron un riesgo relativo de depresión de 8,55 veces más en aquellas que presentaron sobrepeso y obesidad que en las que no tuvieron este desorden de peso; será necesario profundizar en el tema mediante 
nuevos estudios que despejen otro tipo de variables que podrían haber intervenido. La utilización del IDB-IA como indicador de sintomatología depresiva por niveles, en adolescentes, ha sido validada por estudios en otros países, inclusive transculturalmente (Beltrán et al.) y creemos que fue un instrumento efectivo para la investigación desarrollada en esta muestra de mujeres adolescentes quiteñas.

OCAMPO, J.; GUERRERO, M.; ESPÍN, L.; GUERRERO, C. \& AGUIRRE, R. Association between body mass index and depression in adolescent women. Int. J. Morphol., 35(4):1547-1552, 2017.

SUMMARY: Overweight and obesity are persistently increasing worldwide, mainly in adolescents. Both are related to serious chronic diseases and depression, especially in women. We studied 180 adolescent women from the city of Quito, Ecuador. After obtaining informed consent, the women were sampled by two-stage clusters, body mass index (BMI) was measured and Beck's Depression Inventory (BDI 1-A) was performed. The data were entered in Excel 2011 and processed in SPSS v22, to determine the association between BMI and depression. The internal validation of the BDI was calculated and Cronbach's alpha values of 0.818 were obtained. For the response variable, one-way ANOVA test was used, with an alpha error $<0.05$; the GamesHowell post-hoc test was used to differentiate the means; descriptive data of position and dispersion of the variables were obtained. We identified $27.22 \%$ of the individuals with overweight and obesity, and $25.56 \%$ with depression issues, being more frequent in 19year-old women. There was a difference between the means of depression of the normal weight group vs. overweight and obesity $(\mathrm{p}<0.05)$; the post-hoc test of Games-Howell differentiated normal weight-overweight $(\mathrm{p}<0.05)$. The relative risk was 8.55 . These results are similar, both in overweight and obesity variables, and in depression, to those reported in other studies. A statistically significant association was observed between the study variables (overweight-obesity and depression), in adolescent women from the city of Quito, Ecuador.

KEY WORDS: Body mass index; Obesity; Depression; Beck's depression inventory; Adolescents.

\section{REFERENCIAS}

Aguilar Cordero, M. J.; González Jiménez, E.; García García, C. J.; Álvarez Ferre, J.; Padilla López, C. A. \& Mur Villar, N. Estudio comparativo de la eficacia del índice de masa corporal y el porcentaje de grasa corporal como métodos para el diagnóstico de sobrepeso y obesidad en población pediátrica. Nutr. Hosp., 27(1):185-91, 2012.

Beltrán, M. C.; Freyre, M. A. \& Hernández-Guzmán, L. El Inventario de Depresión de Beck: Su validez en población adolescente. Ter. Psicol., 30(1):5-13, 2012

Calderón, C.; Forns, M. \& Varea, V. Implicación de la ansiedad y la depresión en los trastornos de alimentación de jóvenes con obesidad. Nutr. Hosp., 25(4):641-7, 2010.
Camarillo, O. N.; Cabada, R. E; Gómez, M. A. J. \& Munguía, A. E. K. Prevalencia de trastornos de la alimentación en adolescentes. Rev. Esp. Med. Quir., 18(1):51-5, 2013.

Cañoles, C. F.; Araneda, G. N.; Silva, M. H. \& Sanhueza, C. A. Correlation between BMI and depressive symptoms in a sample of preschool children in the Los Rios Region, Chile. Int. J. Morphol., 33(3):860-4, 2015.

Ceja Espíritu, G.; Vizcaíno Orozco, F. J.; Tapia Vargas, R.; Cadenas Freixas, J. L.; Baltazar Rodríguez, L. M. \& Montero Cruz, S. A. Depresión en pacientes de la Unidad de Medicina Familiar 19 con diagnóstico de sobrepeso y obesidad. Rev. Cuba. Invest. Biomed., 29(2):237-43, 2010.

Contreras, A. M. L.; Morán, K. J.; Frez, H. S.; Lagos, O. C.; Marín, F. M. P.; Pinto, B. M. A. \& Suzarte, A. E. Conductas de control de peso en mujeres adolescentes dietantes y su relación con insatisfacción corporal y obsesión por la delgadez. Rev. Chil. Pediatr., 86(2):97-102, 2015.

Fernández-Bustos, J. G.; González-Martí, I.; Contreras O. \& Cuevas, R. Relación entre imagen corporal y autoconcepto físico en mujeres adolescentes. Rev. Latinoam. Psicol., 47(1):25-33, 2015.

Freire, W.; Ramírez-Luzuriaga, M.; Belmont, P.; Mendieta, M.; SilvaJaramillo, M.; Romero, N.; Sáenz, K.; Piñeiros, P.; Gómez, L. \& Monge, R. Tomo I: Encuesta Nacional de Salud y Nutrición de la Población Ecuatoriana de 0 a 59 años (ENSANUT-ECU 2012). Quito, Ministerio de Salud Pública/Instituto Nacional de Estadísticas y Censos, 2014.

Jiménez-Cruz, B. E. \& Silva-Gutiérrez, C. Niveles de ansiedad y depresión tras la exposición breve a imágenes publicitarias en relación con el índice de masa corporal y las actitudes hacia el peso y la alimentación en un grupo de mujeres universitarias. Rev. Mex. Psicol., 25(1):8998, 2008.

Kaufer-Horwitz, M. \& Toussaint, G. Indicadores antropométricos para evaluar sobrepeso y obesidad en pediatría. Bol. Med. Hosp. Infant. Mex. 65(6):502-18, 2008.

Lacunza, A. B.; Caballero, S. V.; Salazar, R.; Sal, J. \& Filgueira, J. Déficits sociales en adolescentes con sobrepeso y obesidad. Cienc. Psicol., $7(1): 25-32,2013$.

Lacunza, A.; Caballero, S.; Salazar, R; Sal, J.; Ale, M.; Filgueira, J.; Fernández, N. \& Díaz, Y. Características de personalidad en adolescentes con sobrepeso y obesidad. Psicol. Salud, 25(1):5-14, 2015.

Martínez Rojano, P. G.; Rojas Russell, M. E.; Serrano Alvardo, K.; López Cervantes, M.; Aedo Santos, A. \& Flórez Alarcón, L. Relación de la actividad física y el peso corporal con sintomatología depresiva en personas con sobrepeso u obesidad. Psicol. Salud, 24(2):187-97, 2014.

Miranda, F. Variabilidad del índice de masa corporal en relación al trastorno depresivo y al trastorno depresivo acompañado de patología cardiovascular. Akadémeia, 4(1):49-60, 2013.

Morla Boloña, R.; Saad de Janon, E. \& Saad, J. Depresión en adolescentes y desestructuración familiar en la ciudad de Guayaquil, Ecuador. Rev. Colomb. Psiquiatr., 35(2):149-65, 2006.

Ortega Becerra, M. A.; Muros, J. J.; Palomares Cuadros, J.; Martín Sánchez, J. A. \& Cepero González, M. Influencia del índice de masa corporal en la autoestima de niños y niñas de 12-14 años. An. Pediatr. (Barc.), 83(5):311-7, 2015.

Padilla, J. Relación del índice de masa corporal y el porcentaje de grasa corporal en jóvenes venezolanos. Rev. Iberoam. Cienc. Act. Fis. Deporte, 3(1):27-33, 2014.

Ponce, G. M.; Quezada, A. O.; Rodríguez, M. A.; Boeri, M. P.; Soto, M. S. \& Brites, F. D. Obesidad por índice de masa corporal y obesidad central en adolescentes de Comodoro Rivadavia, Patagonia Argentina. Rev. Asoc. Latinoam. Diabetes, 22(1):14-21, 2014.

Quintero, J.; Alcántara, M. P. F.; Banzo-Arguis, C.; Martínez de Velasco Soriano, R.; Barbudo, E.; Silveria, B. \& Pérez-Templado Ladrón de Guevara, J. Psicopatología en el paciente con obesidad. Salud Ment., 39(3):123-30, 2016.

Ramos Rosales, J. H.; González Alcántara, K. E. \& Silva, C. Efecto de la interacción entre el sexo y el peso sobre el comer emocional en adolescentes. Psicol. Salud, 26(1):63-8, 2016.

Ríos Saldaña, M. R. \& Osornio Castillo, L. Perfil emocional, obesidad 
OCAMPO, J.; GUERRERO, M.; ESPÍN, L.; GUERRERO, C. \& AGUIRRE, R. Asociación entre índice de masa corporal y depresión en mujeres adolescentes de la ciudad de Quito, Ecuador. Int. J. Morphol., 35(4):1547-1552, 2017.

percibida vs IMC y rendimiento académico en estudiantes universitarios. Altern. Psicol., 17(29):150-64, 2014.

Trejo Ortiz, P. M.; Castro Veloz, D.; Facio Solís, A.; Mollinedo Montano, F. E. \& Valdez Esparza, G. Insatisfacción con la imagen corporal asociada al Índice de Masa Corporal en adolescentes. Rev. Cuba. Enferm., 26(3):150-60, 2010

Yépez, R.; Carrasco, F. \& Baldeón, M. E. Prevalencia de sobrepeso y obesidad en estudiantes adolescentes ecuatorianos del área urbana. Arch. Latinoam. Nutr., 58(2):139-43, 2008.
Dirección para correspondencia:

Juan Emilio Ocampo Bustos

Profesor de la Cátedra de Histología Normal

Carrera de Medicina

Universidad Central del Ecuador

Quito

ECUADOR

Email: j.ocampo01@ufromail.cl

Recibido :30-09-2017

Aceptado:25-10-2017 